\title{
El desaprendizaje: propuesta para profesionales de la salud críticos
}

\author{
Unlearning: Proposal for critic health professionals.
}

\author{
J.L Medina ${ }^{a}$, X. Clèries ${ }^{b}$, M. Nolla ${ }^{c}$ \\ ${ }^{a}$ Universitat de Barcelona, ${ }^{\mathrm{b}}$ Institut d'Estudis de la Salut, ${ }^{\mathrm{c}}$ Fundació Doctor Robert
}

El desaprendizaje como método reflexivo de cuestionamiento crítico de la realidad, ayuda a examinar las estructuras teóricas que cada sujeto ha asimilado, frecuentemente de manera acrítica, durante su trayectoria formativa y profesional. Este examen crítico de las formas habituales de pensar y actuar en el mundo no pretende erigirse como guía del proceso de transición hacia formas de conciencia más verdaderas y fundamentadas. Lo que se busca, por el contrario, es realizar un ejercicio constante de interrogación de lo evidente y de aceptar los límites del pensamiento y las incertidumbres del presente.

Palabras clave: desaprendizaje; pensamiento crítico; complejidad; racionalidad técnica; problemática del saber.

Unlearning as a reflexive method of critical questioning of reality helps us examine the theoretical structures that each individual has mastered, more often than not in an "acritical" manner, during his or her professional and formative trajectory. This critical examination of the habitual ways of thinking and behaving in today's world is not intended, however, to serve as a guide for this transition process towards more authentic and fundamental forms of awareness. On the contrary, what we are looking for is to perform a continuous questioning exercise of what is evident and to accept the limits of our thinking patterns and the uncertainties of our time.

Key words: unlearning, critical thinking, complexity, technical rationality, the problematic of knowledge

Inmersos en una época de globalización, se hace necesario recuperar un pensamiento crítico que permita mirar más allá de lo que se presenta como conocimiento lícito y verdadero. Para evitar el anquilosamiento de las prácticas profesionales, es imprescindible cuestionar las formas habituales de pensarlas, describirlas y llevarlas a cabo. Se impone, pues, la búsqueda de sentidos innovadores y la aproximación a nuevas lógicas que permitan escuchar los márgenes de la maquinaria de las instituciones profesionales y observar a través de sus grietas. Se trata de invertir los horizontes de significados y romper las distintas capas de supuestos sedimentados de forma acrítica durante los procesos institucionales, individuales, sociales e históricos. Para ello, es deseable ejercitar la 
sospecha sobre lo que aparentemente es lógico, coherente y verdadero. En definitiva, desaprender para reconstruir, innovar y transformar las prácticas profesionales, constituye la finalidad que pretende alcanzar la metodología del desaprendizaje.

A menudo se tiene la imagen idealizada del proceso de aprendizaje, en el sentido que se produce de forma creciente y lineal. Basta con observar el propio proceso de desarrollo profesional para darse cuenta que, según Colin Coles ${ }^{1}$, “... El desarrollo profesional no es un proceso lineal. Requiere transformaciones, a veces dolorosas y otras estimulantes, pero que esencialmente implican nuevos insights sobre uno mismo y sobre el propio compromiso con una buena práctica".

Otra fantasía frecuente en el mundo de la educación es pensar que se trata simplemente de añadir conocimiento al que se posee previamente. Esta visión simplificada del proceso de aprender, ignora que para la adquisición de según qué aprendizajes, no basta con la mera adición de contenidos sino que requiere de una transformación del conocimiento. Y la transformación, a menudo precisa deshacer ideas, destruir para reconstruir. En resumen, que a veces, para aprender, primero hay que desaprender.

\section{¿QUÉ ES EL DESAPRENDIZAJE?}

El desaprendizaje es una extrapolación a los campos profesionales de la noción de "deconstrucción" del filósofo francés Jacques Derrida², pensador postmoderno que ha suscitado las más grandes adhesiones y las más virulentas críticas, y se puede entender como un proceso (auto) formativo, individual y/o colectivo de búsqueda de nuevos significados y de sentidos innovadores. El desaprendizaje o deconstrucción es un proceso de inversión de los horizontes de significado que cada sujeto ha sedimentado, en ocasiones, de forma acrítica durante su trayectoria formativa y profesional. Implica examinar críticamente el marco conceptual que estructura la percepción de la realidad y el modo de interpretar el mundo. Desaprender es ejercitar la sospecha sobre aquello que se muestra como "aparentemente lógico", "verdadero y coherente". Es suspender la evidencia de los modos habituales de pensar y describir las prácticas profesionales.

Aplicado en el ámbito de la salud, se cuestiona hasta qué punto los marcos teóricos y disciplinares que fundamentan las acciones cotidianas de los profesionales limitan y amordazan su pensamiento. Desaprender para desarrollar nuevas aproximaciones a la realidad profesional significa desmantelar sus elementos y analizar sus implicaciones para la práctica. Se trata, en suma, de desarrollar un pensamiento reflexivo y crítico el cual permita, mediante el cuestionamiento de las formas de experiencia personal, la reconstrucción, innovación, transformación y mejora de las prácticas.

\section{LAS BASES Y PRINCIPIOS DEL DESAPRENDIZAJE}

Uno de los aspectos más relevantes del desaprendizaje es que permite, partiendo de las propias experiencias, evocar el camino que ha conformado las construcciones personales e identificar las lagunas, los vacíos y las contradicciones de los marcos categoriales. Permite examinar los determinantes sociales y personales que conforman la arquitectura conceptual y perceptual.

El desaprendizaje es, por tanto, un proceso de subjetivación que implica el partir de sí2. Esto significa valorar el propio saber y pensamiento surgido de la experiencia vital y poner en cuarentena los enfoques técnicos que supuestamente tienen una base científica pero ignoran los procesos vitales y la complejidad de los vínculos humanos que se gestan a diario en los contextos de enseñanza y práctica de la atención a la salud. Debe puntualizarse, sin embargo, que el problema no reside en la posible validez de la "teoría", sino en la manera cómo un profesional se enfrenta a ella y cómo influye en su propia práctica. Es decir, a la relación teoría-práctica o, mejor al vínculo entre razón y experiencia. La experiencia se encuentra allá donde lo vivido va acompañado de pensamiento. El saber que procede de la experiencia es el que se mantiene en una relación pensante con las situaciones vividas, es el saber de quien no acepta una forma de entender su trabajo según los criterios de significación dados sino que va en busca de su propia comprensión.

A pesar de que estos procesos de subjetivación pueden producir rechazo e inseguridad al tener que aceptar la ambigüedad y la incertidumbre, constituyen la base de un saber que es consciente de sus límites y de sus imperfecciones, es decir, generador de verdadero conocimiento científico.

Todo este proceso de ruptura, autocrítica y posterior reconstrucción se basa en tres principios epistemológicos que cada vez más están siendo aceptados en los campos teóricos y profesionales del ámbito científico ${ }^{4}$. Esos tres principios implican tres rupturas: con la verdad absoluta, con las miradas únicas y con la racionalidad técnica como única forma de 
conocimiento. La necesidad de esa ruptura viene dada porque esos tres elementos constituyen la simplicidad, unidad y certeza que paralizan y despojan de los nuevos sentidos creados y recreados en las prácticas profesionales.

\section{LA RUPTURA Y/O NEGACIÓN DE LAS VERDADES ABSOLUTAS}

El primer principio del desaprendizaje consiste en suspender el juicio e introducir la duda en la estructura de los pensamientos y representaciones simbólicas. Este trabajo intelectual implica una fuerte crítica a verdades establecidas ${ }^{5}$ y a los modelos "tradicionales "de vida profesional y supone un ejercicio estratégico que permite encontrar las verdades particulares y significativas (el partir de sí al que antes se aludía).

En este principio debe distinguirse, tal y como propone Edgar Morin ${ }^{6}$, entre racionalidad y racionalización. El desaprendizaje aspira a la racionalidad que es el establecimiento de un diálogo con el mundo para intentar hacerlo inteligible, no pretende ser omnipotente ni omnicomprensivo, no es arrogante: la realidad es más rica que lo que las estructuras de nuestro cerebro pueden concebir. Por el contrario, la racionalización es aquel intento quimérico de pretender encerrar la realidad en un único sistema de pensamiento y todo aquello que en la realidad contradice a ese sistema es descartado. El problema de la aspiración a la verdad absoluta (racionalización) es que conlleva la tendencia a explicar de manera simplista aquello que nuestra mente no alcanza a comprender ${ }^{7}$. A través de este principio, sin duda, se aboga decididamente a favor de profesionales reflexivos ${ }^{8,9}$.

\section{LA RUPTURA Y/O NEGACIÓN DE LAS MIRADAS ÚNICAS Y SIMPLIFICADORAS}

El desaprendizaje invita a detenerse, girar la mirada y tomar el propio pensamiento como objeto de análisis, ver desde otro plano, romper con la mirada binaria y disyuntiva del mundo que impide constituir comunidades de sentido que negocien con la heterogeneidad de lógicas y saberes. Supone mirar la realidad desde su complejidad ontológica sin simplificarla, esto es, desde una perspectiva multidimensional, y multidisciplinar. Actualmente resulta erróneo considerar el ser humano como un conjunto material, formado por unidades igualmente materiales, inde- pendientes entre sí como pudieran serlo las "piezas" de cualquier maquinaria mecánica. Se sabe, en cambio, que el ser humano es un producto muy complejo, material - mental, que está formado por múltiples elementos concatenados entre sí y con programas de comportamiento propios y muy complejos constituyendo una individualidad única y distinta de las otras, incluyendo su componente de naturaleza mental integrada con los otros componentes de naturaleza "material". Así por ejemplo, hay que considerar “... el papel central que la biología molecular, la genética, la bioquímica y otras disciplinas aparentemente alejadas de sus intereses primordiales, como las matemáticas, la física moderna, la psicología y la antropología, entre muchas otras, ejercen sobre el saber cardiológico vigente" ${ }^{10}$.

La ruptura de la mirada única implica la necesidad del tránsito del "paradigma de la simplicidad" al de la "complejidad". El paradigma de la simplicidad, en el que la mayoría de profesionales de la salud han sido formados, incurre en una fragmentación reductora del estudio disciplinar del hombre, soslayando, como sostiene Testa, la importancia de las intermediaciones entre fenómenos naturales y fenómenos sociales ${ }^{11}$.

La complejidad surge a principios del siglo XX en la microfísica, pero al ser ésta considerada un caso límite, se olvidó que esa frontera conceptual concernía a todos los fenómenos materiales, incluidos los de nuestro propio cuerpo y cerebro ${ }^{6}$. Pero, ¿qué es esa complejidad que permite y exige una multiplicidad de miradas? Para Morin ${ }^{12}$, es un fenómeno cuantitativo, una cantidad extrema de interacciones e interferencias en un número muy grande de unidades. Por ejemplo, el ser humano combina billones de células. Además de estas interacciones cuantitativas que superan nuestra capacidad de cálculo, la complejidad comprende también incertidumbres, indeterminaciones y fenómenos aleatorios. La complejidad siempre está relacionada con el azar. Cuando emprendemos una acción ésta entra en un universo de interacciones que hacen que escapen a nuestra intención primera. La acción humana requiere estrategia que no es lo mismo que programa. Esta misma afirmación es válida para las acciones que tratan de anticipar los cursos de acción en las prácticas profesionales de la salud ${ }^{13}$.

El desaprendizaje conlleva el abandono de la lógica disyuntiva, hipotético-deductiva y lineal, sustituyéndola por la lógica dialéctica de la complejidad. Se trata de unir dos nociones que lógicamente parecen excluirse: los principios antagónicos cooperan para 
generar estructura. Es decir, la contradicción o la paradoja no son errores del pensamiento (como había enseñando la lógica aristotélica) sino que son muestras de las capas profundas de los fenómenos sociales y biológicos que son irreductibles a la lógica. Un ejemplo de la fisiología nutricional puede ilustrar cómo la paradoja nos constituye. El desequilibrio nutricional permite al sistema mantenerse en equilibrio (estabilidad y continuidad de los procesos orgánicos), el cual se degrada si hay clausura del sistema. Las estructuras (órganos) se mantienen mientras los elementos (células) cambian. Las células se renuevan mientras que el conjunto permanece estable. Para ello el sistema debe cerrarse al mundo exterior a fin de mantener el medio interno que si no se degradaría (hemorragia). Pero su apertura (alimentación) es lo que permite su clausura (estado intacto de la piel).

En resumen, se trata de mirar la realidad profesional de manera ampliada y más respetuosa para con su dinamismo, ambigüedad, inestabilidad e indeterminación. Pasar de visiones simples a visiones complejas rompiendo con los reduccionismos empobrecedores.

\section{LA RUPTURA Y/O NEGACIÓN DE LA RACIONALIDAD TÉCNICA COMO ÚNICA FORMA DE CONOCIMIENTO}

La racionalidad técnica ha sido dominante en la investigación, la práctica educativa y la formación de profesionales de la salud en los últimos 50 años. La concepción de la asistencia como intervención técnica, la ubicación de la investigación en los métodos cuantitativos y la formación inicial dentro del modelo de entrenamiento basado en competencias son manifestaciones de su hegemonía. Esta perspectiva, en la que todavía son educados la mayoría de profesionales, surge como intento de reivindicar un estatus y rigor de los que carecía la práctica tradicional, considerándola como una ciencia aplicada. El fundamento epistemológico de esta concepción es lo que se ha denominado la racionalidad técnica. En su desarrollo histórico podemos encontrar dos modelos que suponen elaboraciones progresivas en la manera de entender la relación entre el conocimiento académico y la práctica profesional: el modelo de entrenamiento y el de resolución de problemas.

Sin embargo, desde mediados del siglo XX, se ha venido realizado una crítica generalizada a la racionalidad técnica desde diferentes ámbitos científicos ${ }^{14,15}$ $\mathrm{y}$ académicos ${ }^{16}$, que ha dado lugar a una serie de concepciones de la práctica profesional en el ámbito de la salud y de la formación de profesionales que abogan por la necesidad de analizar y comprender lo que realmente hacen profesionales cuando se enfrentan a las situaciones, singulares, inestables y ambiguas de su práctica $^{17,18}$. Desde esta perspectiva se defiende que la actividad del profesional tiene lugar en situaciones sociales de gran complejidad y ambigüedad que no pueden resolverse por la mera aplicación de conocimientos científicos, ya que el mundo de la asistencia a la salud es demasiado fluido y reflexivo para permitir tal sistematización y los problemas no aparecen bien delimitados ni siquiera claramente definidos.

\section{EL PROCESO DE DESAPRENDIZAJE}

El desaprendizaje como proceso no tiene final y como forma no es lineal sino espiral. Es un ciclo de nueve momentos que, una vez conocido, se va repitiendo de manera constante y se conforma en la manera de pensar y actuar del sujeto reflexivo. En la tabla 1 se describen los diferentes momentos de la metodología del desaprendizaje y las pautas de reflexión recomendadas.

\section{Primer Momento: Reconocimiento de la realidad y definición del aspecto a desaprender}

El proceso de desaprendizaje-aprendizaje se inicia con el reconocimiento explícito de la realidad profesional y su contexto que se quiere abordar y que en muchas ocasiones es motivo de insatisfacción. Este reconocimiento se plantea como el momento de análisis del contexto teórico, práctico, institucional y personal en el cual el profesional está inmerso con el fin de ir identificando las posibles lagunas, inconsistencias, falacias y contradicciones del ejercicio profesional.

A partir de este análisis, emerge la necesidad de deconstruir ciertos aspectos de la práctica, definiendo individual y/o colectivamente lo que se quiere transformar, al nivel de las prácticas profesionales, los saberes que las fundan y los sujetos que las desarrollan. En ocasiones no es fácil tomar conciencia de la necesidad de deconstruir, ni identificar aquellos aspectos de la práctica que deben ser desaprendidos, aunque frecuentemente estas prácticas se manifiestan en forma de conflicto institucional o como acciones organizacionales sin sentido. La elección del aspecto a desaprender está ligada a las características de la institución, a las prácticas profesionales y a los intereses y necesidades de sus protagonistas. Las pautas de reflexión que pueden ayu- 
dar a identificar el aspecto o temática a desaprender aparecen en la tabla 1.

Es importante remarcar que desaprender no es incorporar nuevos esquemas a los marcos teóricos y de comprensión que utiliza el profesional, sino examinar críticamente la propia acción profesional estableciendo una relación reflexiva permanente con la práctica, esto es, entender nuestro conocimiento como problemático. Ello significa que el conocimiento profesional se halla en dependencia constante de unos marcos de interpretación que pueden y deben ser sometidos a crítica y objeto de transformación.

Por último, recalcar que el desaprendizaje se concreta en las personas que deseen ser parte de la experiencia y que entienden que existen aspectos de su práctica susceptibles de mejora. Este proceso personal-intelectual no puede ser entendido como un experimento para concluir al final del proceso como una experiencia interesante sin más, sino como la decisión de una búsqueda reflexiva para transformar la práctica profesional.

\section{Segundo Momento: La identificación de las "huellas " personales.}

El segundo momento del desaprendizaje consiste en una actividad biográfico-reflexiva eminentemente individual y personal. Se trata de reflexionar sobre la experiencia personal (intelectual, práctica, emocional) con relación al elemento que está siendo deconstruido. Esa reflexión retrospectiva tiene como finalidad desvelar la forma cómo se ha ido conformando y construyendo en uno mismo (experiencias formativas, profesionales, etc.) el aspecto a desaprender.

Se parte, en primer lugar, de la elaboración de la "huella personal", que no es otra cosa que la reflexión escrita sobre la historia individual con relación al elemento a desaprender. Esta historia se constituye tanto en el punto de partida como en el contexto de "inteligibilidad" del tema a desaprender.

\section{Tabla 1. Metodología del desaprendizaje (propuesta de Medina, 2003)}

\begin{tabular}{ll}
\hline Momentos & Pautas de reflexión \\
\hline Primer Momento: Reconocimiento de la realidad & $\bullet$ ¿Qué aspectos de mi práctica me generan insatisfacción? ¿En qué \\
y definición del aspecto a desaprender & teorías o modelos se apoyan esos aspectos? ¿Existen otras teorías o \\
& modelos alternativos? \\
& $\bullet$ ¿Hay algún aspecto de los esquemas teóricos que manejo con los \\
& que me siento insatisfecho o considere poco útiles para mi práctica? \\
& ¿Cómo los aprendí? ¿Cómo los uso? ¿Son "evidentes" las evidencias \\
& en las que me baso para tomar decisiones profesionales?
\end{tabular}

Segundo Momento: La identificación de las "huellas" personales
- ¿Cómo se presenta en mí y entiendo hoy el/la aspecto a desaprender (cómo lo entiendo, cómo lo percibo, qué conozco de él: certezas y dudas)?

- Cómo ha sido construido en mí el/la aspecto a desaprender (experiencia formativa, lecturas, práctica profesional, etc.).

Tercer momento: Elaboración del

- Identificar elementos y componentes de lo que quiero deconstruir

- Búsqueda de información bibliográfica (documentos primarios, secundarios, bases de datos, índices, etc.)

- Consulta a expertos

- Sistematización conceptual

Quinto Momento: El des-aprendizaje (desandamiaje)

- ¿Qué elementos o enfoques nuevos incorporo a mis concepciones? - ¿Qué aspectos ya conocidos desestimo? ¿Cuáles mantengo?

Sexto Momento: Planificación de la práctica transformadora; inicio de la reconstrucción

Séptimo Momento: Seguimiento de las acciones

Octavo Momento: Retorno a la realidad

transformada; Realidad II
- ¿Qué aspectos voy a transferir a mi práctica profesional?

- ¿Mediante que procesos y procedimientos?

- ¿Cómo evaluaré los cambios y transformaciones operadas?

- ¿Qué espacios y momentos serán usados para la evaluación?

- ¿Cómo percibo ahora mi realidad profesional?

- ¿Qué fortalezas y debilidades hay en las transformaciones que se están operando?

Noveno Momento: El inicio de un nuevo desaprendizaje.
- ¿Qué nuevos aspectos de mi práctica requieren ser deconstruidos? 
La elaboración escrita de la huella personal significa la producción propia en relación con el aspecto que se desaprende y desde el cual se construye "el propio texto". En este sentido, cada sujeto, partiendo de sí, reconstruye de manera escrita su experiencia particular a través de una autobiografía, la cual puede articularse en torno a las cuestiones apuntadas en la tabla 1.vEstas preguntas, que pueden ser formuladas de diferente manera en los distintos contextos en los que se aplican, permiten visualizar las contradicciones, falacias y lagunas en los sistemas apreciativos y las representaciones de poder $^{19}$ que se encuentran presentes en cada uno de los profesionales.

\section{Tercer momento: Elaboración del}

"mapa" individual y/o colectivo.

Dado que el desaprendizaje busca la "transformación" de la teoría, la práctica y los imaginarios colectivos, en este tercer momento deben identificarse los elementos y componentes que conforman el factor que está siendo deconstruido (mapa personal). Aunque es un momento que debe ser desarrollado colectivamente, a efectos formativos su aprendizaje también puede contemplarse individualmente. Estos elementos deberán ser compartidos entre los integrantes del grupo (cuando los miembros del grupo ejercen en una misma institución y desarrollan funciones similares) para elaborar el mapa colectivo.

Este mapa "institucional" refleja la comprensión que los sujetos tienen del elemento a desaprender. Es la diagramación de cómo se presenta en la institución ese elemento (saberes, prácticas, relaciones formales e informales, etc.). En este momento se pasa del "propio texto" (la huella) a la intertextualización reconociendo e integrando las interpretaciones del resto de miembros del grupo (lo idóneo es formar grupos heterogéneos no muy numerosos para favorecer una visión lo más amplia posible del elemento a desaprender). Algunas preguntas que pueden facilitar la elaboración del mapa individual y/o colectivo, aparecen en la tabla 1.

Es importante destacar que la elaboración del mapa colectivo se desarrolla en un proceso intenso de negociación, en el cual las preguntas anteriores ayudan a desvelar los saberes, las prácticas y las relaciones en las instituciones. Ese mismo análisis permite, además, vislumbrar de manera heurística algunas de las posibilidades potenciales de transformación de la práctica, las cuales son sistematizadas en el siguiente momento.

\section{Cuarto Momento: La búsqueda de "interpretaciones- comprensiones-acciones" alternativas.}

Este momento del proceso posibilita la confrontación de la práctica personal/institucional, articulada en el mapa individual/colectivo, con otras explicaciones/ interpretaciones del tema a desaprender elaboradas a lo largo de la historia. Se trata de presentar a los miembros del grupo las teorías, enfoques o modelos alternativos que se han ido dando a lo largo del tiempo sobre el elemento que está deconstruyendo. La finalidad es conocer "otras" interpretaciones y examinar su pertinencia y validez para hacer "inteligible" el elemento a desaprender, y su relevancia para la práctica profesional. En suma, "pensar" la práctica a la luz de otras miradas y posibilidades de comprensión que vayan más allá de la ortodoxia dominante. Para lograr esta finalidad existen diversas estrategias que pueden ser realizadas individual o grupalmente (ver tabla 1 ).

\section{Quinto Momento: El des-aprendizaje (desandamiaje).}

Una vez elaborada la autobiografía acerca de la temática (huella), identificados los elementos de esa temática que están presentes (en distintas formas) en la práctica profesional y las teorías alternativas, tiene lugar el desaprendizaje propiamente dicho. En este momento se desarrolla un análisis colectivo tanto de lo elaborado en el momento tercero (el mapa) como en el cuarto (las alternativas). Se trata de identificar/priorizar (desmantelar) en el mapa aquellos elementos considerados no pertinentes (obsoletos, erróneos, poco útiles,...) y los que pueden mantenerse; así como los considerados imprescindibles procedentes del análisis realizado en el momento anterior.

Una analogía útil puede ser la del arquitecto que trata de construir una casa nueva a partir de una antigua. Analiza los elementos de la casa vieja (el mapa elaborado en el tercer momento) y determina las estructuras que no servirán y aquéllas que podrán tener todavía utilidad en la nueva construcción ya sea modificándolas o manteniéndolas. También analiza los nuevos materiales de que dispone (las alternativas teóricas) y decide cuáles utilizará. Algunas preguntas que pueden orientar este proceso colectivo se pueden observar en la tabla 1 .

Este es un momento de una gran intensidad creativa y productiva en el plano intelectual-personal y que abre la puerta al momento siguiente en el que se decide acerca de los cursos de acción futuros. Aquí el nuevo aprendizaje (la casa nueva) se funda sobre lo ya conocido y dado. 
Tabla 2. Características del proceso de socialización de los médicos en formación

- Simplificación y estandarización de la práctica médica.

- Tendencia a eliminar la complejidad y la incertidumbre.

- Prevalencia de la estandarización en detrimento de la singularidad del paciente.

- Sobrevaloración del aprendizaje biomédico.

El nuevo aprendizaje se basa en el desaprendizaje, el abandono de ciertas concepciones teórico-prácticas (la destrucción de los ladrillos inservibles para soportar la nueva estructura, siguiendo la analogía), en el mantenimiento de aquello que aún es válido y de lo novedoso que vale la pena ser incorporado (los nuevos materiales). Este "desandamiaje" es el primer paso de esta fase "reconstructiva" del proceso.

\section{Sexto Momento: Planificación de la prácticatransformadora; inicio de la reconstrucción.}

A partir de aquí es necesario concretar en acciones todo lo desaprendido-aprendido (métodos, procesos, protocolos, procedimientos, estrategias, etc). Se trata de elaborar un plan o agenda de trabajo en las diferentes dimensiones, áreas de intervención $\mathrm{u}$ otras identificando responsabilidades y concreciones reales y posibles.

\section{Séptimo Momento:}

\section{Seguimiento de las acciones.}

Este momento es importante porque permite "modular" y "contextualizar" las acciones previstas en función de las situaciones que vayan surgiendo. Es decir, todo el proceso de desaprendizaje no puede quedar en una simple transformación de las comprensiones o en una ganancia cognitiva, sino que debe "materializarse" en nuevas acciones. Esta "nueva" práctica deberá ser parte de los nuevos proyectos, de las propuestas y concreciones de las mismas, exigiendo permanentes momentos de evaluación y ajuste para lograr concretar los nuevos aprendizajes y transformaciones.

El seguimiento supone la creación de momentos y espacios (recogidos en la planificación anterior) para analizar las contrariedades y fortalezas de la nueva práctica. De ese modo, estos espacios son momentos de consolidación y mejora de toda la experiencia de desaprendizaje.

\section{Octavo Momento: Retorno a la realidad transformada; realidad II.}

El retorno a la realidad profesional "desaprendi- da" se hace ahora desde una posición conceptual y perceptiva "cualitativamente" diferente a la situación de inicio del proceso.

Es importante remarcar que este retorno no debe ser entendido como el final del proceso sino como un momento de un continuo aprendizaje-desaprendizaje-aprendizaje, es decir, como una fase de un proceso de cambio y transformación permanente.

\section{Noveno Momento: El inicio de un nuevo desaprendizaje.}

La reconstrucción y mejora de las prácticas profesionales son, en realidad, procesos inacabados y de búsqueda permanente que afectan constantemente a los saberes y las prácticas sedimentadas y consolidadas. En este sentido, la lógica reflexiva y reconstructiva que el desaprendizaje supone, forma ya parte de los modos perceptuales, los estilos cognitivos y las formas de trabajo del profesional, convirtiéndose a sí en un profesional "crítico-reflexivo.

\section{CONSIDERACIONES}

A pesar de tratarse de una interesante e innovadora metodología, la aplicación del desaprendizaje genera más preguntas que respuestas, apuntándose como una manera más sistemática de potenciar el autoconocimiento que otras propuestas reflexi$\operatorname{vas}^{20,21}$. También constituye una forma más constructiva -y madura- de afrontar las numerosas fuentes de insatisfacción en el ámbito sanitario que instalan a algunos profesionales en un estado de queja permanente y abonan la aparición del tan cacareado estrés laboral o burnout, intensamente aireados en los espacios informales (pasillos, bares, etc.) de los centros y servicios de salud.

Por otro lado, hay que tener presente que la idea de ciencia y del método científico se ha basado en las ciencias naturales de la época de la revolución científica (siglo XVIII), dónde el conocimiento derivado de los hechos de la experiencia, la observación como intervención práctica, el experimento y la inferencia de teorías a partir de los hechos constituían la plataforma esencial de lo que se denomina ciencia ${ }^{22}$. Sin 
embargo, la emergencia del falsacionismo, las aportaciones de Kuhn a la sucesión de paradigmas, el punto de vista bayesiano y, sobre todo, las aportaciones de la mecánica cuántica y la teoría del campo cuántico, entre otras, plantean retos cualitativamente nuevos y múltiples cuestiones a desaprender en el ámbito de la salud.

En este sentido, cabe destacar las conclusiones del estudio de Sánchez et al. ${ }^{23}$ sobre el proceso de socialización de los médicos en formación, planteando elementos susceptibles de ser desaprendidos (tabla 2). Aunque también se pueden apuntar otros ámbitos para aplicar la metodología del desaprendizaje y contribuir a la valoración crítica del sistema sanitario, en algunos casos ya iniciada por diversos autores:

- La deconstrucción de la historia de los saberes sanitarios $^{24-25}$.

- La deconstrucción de los modelos y enfoques de la atención de salud ${ }^{26-29}$.

- La deconstrucción de la epistemología de los saberes sanitarios ${ }^{18,1}$.

- Las razones, intereses, hegemonía y poder del profesionalismo $0^{2,3}$.

\section{BIBLIOGRAFÍA}

1. Coles C. Enfoque del desarrollo profesional. Educación Médica 1998; 1:101-107

2. Derrida J. La deconstrucción en las fronteras de la filosofía: la retirada de la metáfora. Barcelona: Paidós; 1989.

3. Contreras J. La didáctica y la autorización del profesorado. Universidad de Barcelona : Departamento de Didáctica y Organización Educativa; 2002.

4. Horgan J. El fin de la ciencia. Los límites del conocimiento en el declive de la era científica; 1998.

5. López S, Recio H. Creatividad y pensamiento crítico, México: Trillas; 1998.

6. Morin E. Ciencia con conciencia. Barcelona: Anthropos; 1984.

7. Horkheimer M. Crítica de la Razón instrumental. Buenos Aires: SUR; 1969.

8. Schön DA. The reflective practitioner: how professionals think in action. New York: Basic Books; 1983.

9. Shön DA. Educating the reflective practitioners: towards a new design for teaching and learning in the professions. San Francisco: Jossey-Bass; 1987.

10.Bochatay L. et.al. Cardiología transdiciplinaria. Revista Argentina de Cardiología 2001; 30: 660-663.

11.Testa M. Pensar en salud (p. 48). Buenos Aires: Lugar Editorial; 1997

12.Morin E. Introducción al pensamiento complejo. Barcelona: Gedisa; 1995.

13.Medina JL. Algunas reflexiones en torno a la naturaleza com- pleja de la planificación de la enseñanza universitaria: lógica de la formulación versus lógica de la ejecución. Universidad de Barcelona: Departamento de Didáctica y Organización Educativa; 2002.

14.Feenberg A. Critical Theory of Technology. New York: Oxford University Press; 1964.

15.Habermas J. Ciencia y técnica como "ideología". Madrid: Tecnos; 1984.

16. Ferrada D. La diversidad de racionalidades en las distintas acciones pedagógicas. Revista de Ciencias de la Educación, 2001; 186: 159-182.

17.Gadamer HG. El estado oculto de la salud. Barcelona: Gedisa; 1996.

18.Testa, M. Pensar en salud. Buenos Aires: Lugar Editorial; 1997. 19. Medina JL. Saber y poder en la investigación sobre la salud. Universidad de Barcelona: Departamento de Didáctica y Organización Educativa; 1994.

20.Novack DH, Kaplan C, Epstein RM, et al. Personal awareness and professional growth: a proposed curriculum. Med Encounter 1997;13: 2-7.

21.Epstein RM. Mindful practice. JAMA 1999, 282:833-839.

22. Chalmers A. ¿Qué es esa cosa llamada ciencia? Madrid: Siglo XXI de España editores; 2000.

23.Sánchez M. El procés de socialització del personal mèdic intern resident: aportacions de la investigació social qualitativa a l'anàlisi de la iniciació professional en un context institucional. IES, Monografies; 10. Barcelona: Departament de Sanitat i Seguretat Social, Generalitat de Catalunya; 2002.

24.Foucault M. Nacimiento de la Clínica. Mexico: Siglo XXI; 1996. 25.Foucault M. Historia de la medicalización. En: Varela, J. y Uría, F. (Eds.). La vida de los hombres infames. Ensayos sobre desviación y dominación. Madrid: La Piqueta, pp. 121-152; 1990.

26.Airhihenbuwa C. Health promotion and the discourse on culture: implications for empowerment. Health Education Quarterly 1994; 21 (3): 345-353.

27.Comelles J M. La utopía de la atención integral de salud. Autoatención, práctica médica y asistencia primaria. En: Porta, M. y Alvarez-Dardet, C. (Eds.). Revisiones en Salud Pública (Vol. 3 ). Barcelona: Masson, pp. 169-192; 1993.

28.Eakin J, Mclean M. A critical perspective on Research and Knowledge Development in Health Promotion. Canadian Journal of Public Health 1992; 83 (Supl.1): 72-76.

29.Lupton D. The Imperative of Health. Public Health and the Regulated Body. London: Sage; 1995.

30.Almeida-Filho N. La ciencia tímida. Ensayos de deconstrucción de la epidemiología. Buenos Aires: Lugar editorial; 2000.

31. Sykes G. En defensa del profesionalismo docente como una opción de política educativa. Educación y Sociedad 1992; 11: 85-96.

32.Larson M. (1989). Acerca de los expertos y las profesiones o la imposibilidad de haberlo dicho todo. Revista de Educación 1989; No Extraordinario. La comparación en Ciencias Sociales y en Educación: 199-225. 